OPEN ACCESS

Edited by:

Bin Liu,

Nankai University, China

Reviewed by:

Peng Li,

Chinese Center for Disease Contro and Prevention, China

Songzhe Fu,

Dalian Ocean University, China

*Correspondence:

Qinghua Hu

huqinghua03@163.com

Specialty section: This article was submitted to

Clinical Microbiology,

a section of the journal

Frontiers in Cellular

and Infection Microbiology

Received: 14 August 2020 Accepted: 18 January 2021

Published: 26 February 2021

Citation:

Lu L, Li M, Li Y, Jiang M, Jiang Y, Shi X, Zuo L, Wang L, Bian S, Qiu Y, Cai R, Liao Y, Li Q, Li L and Hu Q (2021) A Novel Molecular Method for Simultaneous Identification of Vibrio parahaemolyticus $57 \mathrm{~K}$-Serogroups Using Probe Melting Curve Analysis. Front. Cell. Infect. Microbiol. 11:594808. doi: $10.3389 /$ fcimb.2021.594808

\section{A Novel Molecular Method for Simultaneous Identification of Vibrio parahaemolyticus 57 K-Serogroups Using Probe Melting Curve Analysis}

\author{
Linying $L u^{1}$, Minxu $L^{2}{ }^{2}$, Yinghui $L_{i}^{2}$, Min Jiang ${ }^{2}$, Yixiang Jiang ${ }^{2}$, Xiaolu Shi ${ }^{2}$, Le Zuo ${ }^{2}$, \\ Lei Wang ${ }^{2}$, Shengzhe Bian ${ }^{3,4}$, Yaqun $\mathrm{Qiu}^{2}$, Rui Cai ${ }^{2}$, Yiqun Liao ${ }^{5}$, Qingge $\mathrm{Li}^{5}$, Liqiang $\mathrm{Li}^{4}$ \\ and Qinghua $\mathrm{Hu}^{1,2 *}$

\begin{abstract}
'School of Public Health, University of South China, Hengyang, China, ${ }^{2}$ Shenzhen Center for Disease Control and Prevention, Shenzhen, China, ${ }^{3}$ School of Public Health (Shenzhen), Sun Yat-sen University, Guangzhou, China, ${ }^{4}$ Shenzhen Key Laboratory of Unknown Pathogen Identification, BGI-Shenzhen, Shenzhen, China, 5 School of Life Sciences, Xiamen University, Xiamen, China
\end{abstract}

The serotyping of Vibrio parahaemolyticus, which is crucial to the surveillance and detection of outbreaks of vibriosis infection, has been widely used in many countries. In this study, we developed a molecular assay, named multiplex ligation reaction based on probe melting curve analysis (MLMA), for simultaneous identification of $V$. parahaemolyticus $57 \mathrm{~K}$-serogroups. Based on the previous genomes of 418 strains including $39 \mathrm{~K}$-serogroups and the $18 \mathrm{~K}$-serogroups sequences from public databases, we obtained $57 \mathrm{~K}$-serogroups specific gene sequences for designing primers and probes. The developed MLMA assay for identifying the $V$. parahaemolyticus $57 \mathrm{~K}$-serogroups showed high reproducibility, with the intra- and inter-assay standard deviations and coefficients of variation of no more than $1{ }^{\circ} \mathrm{C}$ and $1 \%$, respectively. The limit of detection for all gene targets ranged from 0.1 to $1.0 \mathrm{ng} / \mu \mathrm{l}$. We validated the MLMA assay with a doubleblind test identifying $595 \mathrm{~V}$. parahaemolyticus isolates using conventional serotyping methods for comparison. The results showed the kappa value between the MLMA assay and the traditional serological method was 0.936 and that there was a $96.97 \%$ consistency rate with conventional serotyping methods for all detected isolates. Additionally, five rare K-serogroups were identified using the MLMA assay, as well as 18 strains that could not be identified using the traditional serotyping method. Thus, the MLMA assay provides a rapid, robust, and promising tool for the molecular serotyping of $V$. parahaemolyticus K-serogroups and has the potential application to the detection of outbreaks and surveillance of $V$. parahaemolyticus infection.

Keywords: Vibrio parahaemolyticus, molecular identification, K-serogroups, vibriosis surveillance, multiplex ligation reaction based on probe melting curve analysis 


\section{INTRODUCTION}

Vibrio parahaemolyticus is a Gram-negative, motile bacterium commonly found in marine and estuarine environments worldwide (Letchumanan et al., 2014; Wang et al., 2015). Investigation of $V$. parahaemolyticus infection has revealed that raw or undercooked seafood contaminated by $V$. parahaemolyticus strains is commonly responsible for acute gastroenteritis, and that in rare cases such as wound infection, ear infection or septicemia (Letchumanan et al., 2014). Between 2011 and 2016, 790 outbreaks of foodborne diseases were reported in more than 703 hospitals of China, causing 13,013 individuals to become ill. Of these cases, $V$. parahaemolyticus was the most common pathogen being responsible for foodborne disease outbreaks that caused most cases reported $(42.3 \%)$. Therefore, $V$. parahaemolyticus is recognized as the leading cause of foodborne disease in China (Liu et al., 2018). Recent studies have found that pandemic $V$. parahaemolyticus strains are responsible for approximately 50\%$80 \%$ of all $\mathrm{V}$. parahaemolyticus cases in many countries, including India, Peru, Mexico and Chile (Han et al., 2019). In addition, a newly emerging shrimp disease, acute hepatopancreatic necrosis disease (AHPND), is known to be caused by strains of $V$. parahaemolyticus that contain a unique virulence plasmid. It has caused significant economic loss in global shrimp industry (Lai et al., 2015). Recent genomic analysis of $233 \mathrm{~V}$. parahaemolyticus strains isolated from diseased shrimp, humans and environmental samples suggested that the spread of AHPND is mainly via horizontal transfer of the AHPND-associated plasmid, which highlighted a significant transmission route of $V$. parahaemolyticus between aquaculture and human communities (Fu et al., 2020). Thus, V. parahaemolyticus has become a large public health issue on a global scale, especially in coastal regions (Han et al., 2016).

$V$. parahaemolyticus is a multiserotype bacterium found in foodborne diseases or external environments that includes at least 13 O-serogroups and $71 \mathrm{~K}$-serotypes (Iguchi et al., 1995; Bhuiyan et al., 2002). In 1996, the epidemiology of $V$. parahaemolyticus displayed a radical change with a new serotype, O3:K6, that appeared abruptly (Nair et al., 2007; Baker-Austin et al., 2018). The pandemic O3:K6 strains have specific genetic markers such as positivity for the thermostable direct hemolysin $(t d h)$ gene, negativity for the TDH-related hemolysin (trh) gene and positivity for a toxRS/new gene (Matsumoto et al., 2000; Han et al., 2016). Since then, the serotype O3:K6 and its serovariants have spread rapidly throughout the world (Han et al., 2016). Additionally, a new emerging serotype with enhanced acid resistance, O4:KUTrecAin, has become the second most common serotype following O3:K6. In China, O4:KUT-recAin has become widespread during a short period of time (Chen et al., 2020). Accordingly, serotyping has been widely applied to provide a vital information for epidemiological investigation.

Serotyping is an essential method for identification of pathogens, foodborne disease outbreak investigations and source tracing that has been widely used to obtain an accurate biological phenotype of many pathogens. The serotyping of $V$. parahaemolyticus is generally accomplished using traditional methods based on agglutination with certain antisera. However, serological methods are limited in that they are time-consuming, expensive and labor-intensive. As understanding of antigens and their gene clusters has improved, some molecular approaches have been developed as alternatives to the traditional method. For example, polymerase chain reaction (PCR) assays are often used to identify and distinguish specific serotype. In 2012, an Oserogroup-specific PCR based assay was first used for the identification and detection of $V$. parahaemolyticus pathogens in clinical and environmental samples (Chen et al., 2012). Later, the use of the matrix-assisted laser desorption/ionization time-offlight mass spectrometry (MALDI-TOF MS) enabled detection of a specific serotype, O4:K8, but failed to identify other serotypes (Li et al., 2018). Recently, a microsphere-based suspension array was established for the detection and identification of $55 \mathrm{~V}$. parahaemolyticus K-serogroups based on specific genes of the capsular polysaccharide (CPS) gene cluster (Pang et al., 2019). However, these methods are limited in that they require laborintensive post-PCR manipulations, expensive instruments, or involve the preparation of microsphere beads, which are complicated and time-consuming.

Multiplex ligation reaction based on probe melting curve analysis (MLMA) is a high-throughput, high-accuracy, low-cost method that has been successfully used for the simultaneous identification of 10 bacterial pathogens and applied to the simultaneous identification of 30 common Salmonella serotypes (Jiang et al., 2017; Zuo et al., 2019). In MLMA, a fluorescence signal and melting temperature (Tm) are combined and used as a virtual 2D label that enables homogenous detection of one order of magnitude more targets (Liao et al., 2013). Previously, we presented a molecular assay based on the principles of MLMA to simultaneously identify the 11 clinically most common $V$. parahaemolyticus serotypes ( $\mathrm{Li}$ et al., 2019). In this study, to further increase the number of target $\mathrm{K}$-serogroups in multiplexity, we established a three-tube system to serotype $V$. parahaemolyticus $57 \mathrm{~K}$-serogroups that could be applied as an alternative for the surveillance and control of vibriosis infections.

\section{MATERIALS AND METHODS}

\section{Bacterial Strains}

There were 3,826 strains stored that were isolated from the stool specimens of infectious diarrheal patients and food samples over 15 years (2003-2018) in Shenzhen Center for Disease Control and Prevention, 3,590 strains were K-serogroups typeable and 236 strains were K-serogroups untypeable (KUT). Among 3,826 strains, 418 strains were previously sequenced (Li et al., 2019; Bian et al., 2021). Among 418 strains, 338 strains were selected to develop the MLMA assay (Table 1), which included 328 strains representing $39 \mathrm{~K}$-serogroups and 10 strains of untyped $\mathrm{K}$ serogroups for detection of cross-reactions. In addition, 18 rare K-serogroups were incorporated into the assay using Escherichia coli TOP10 strains $(\mathrm{n}=18$, Table S1) containing serogroupspecific genes cloned into a pUC57 vector (Sangon Biotech Co. Ltd., Shanghai, China). Among 3,826 strains, 595 strains which included $10 \%$ of 3,590 K-serogroups typeable strains $(n=359$, 
TABLE 1 | Reference isolates $(n=338)$ representing $V$. parahaemolyticus K serogroups over a 12-year period (2006-2018) for the development of multiplex ligation reaction based on probe melting curve analysis (MLMA) assay.

\begin{tabular}{|c|c|}
\hline Serotype & Number of isolates \\
\hline $\mathrm{K} 1$ & 3 \\
\hline K3 & 10 \\
\hline K4 & 6 \\
\hline K5 & 1 \\
\hline K6 & 20 \\
\hline K8 & 20 \\
\hline K9 & 20 \\
\hline K11 & 6 \\
\hline K12 & 4 \\
\hline K13 & 11 \\
\hline $\mathrm{K} 17$ & 11 \\
\hline K18 & 3 \\
\hline K19 & 4 \\
\hline K20 & 10 \\
\hline K21 & 2 \\
\hline K23 & 2 \\
\hline K25 & 20 \\
\hline K28 & 9 \\
\hline K29 & 20 \\
\hline K30 & 3 \\
\hline K31 & 1 \\
\hline K32 & 14 \\
\hline K33 & 6 \\
\hline K34 & 10 \\
\hline K36 & 20 \\
\hline K37 & 2 \\
\hline K38 & 2 \\
\hline K41 & 9 \\
\hline K42 & 7 \\
\hline K44 & 8 \\
\hline K48 & 1 \\
\hline K49 & 1 \\
\hline K55 & 3 \\
\hline K56 & 20 \\
\hline K60 & 7 \\
\hline K63 & 4 \\
\hline K68 & 20 \\
\hline K69 & 7 \\
\hline K71 & 1 \\
\hline KUT & 10 \\
\hline
\end{tabular}

Table S2) and all 236 untypeable strains (Table S3) were selected to validate the MLMA assay.

\section{Bacterial Culture, DNA Extraction, and Conventional Serotyping}

All $V$. parahaemolyticus strains were revived and cultured in Vibrio chromogenic agar (Guangdong Huankai Microbial Science and Technology, Guangzhou, China) at $37^{\circ} \mathrm{C}$ for $12 \mathrm{~h}$ to isolate single colonies. Individual colonies were then selected and cultured in $3 \mathrm{ml}$ of alkaline peptone water (Guangdong Huankai Microbial Science and Technology), after which they were incubated at $37^{\circ} \mathrm{C}$ for $16 \mathrm{~h}$ while shaking at $200 \mathrm{rpm}$. Escherichia coli TOP10 strains were cultured in normal LB medium under the same conditions. Genomic DNA templates were extracted using the boiled lysates method, in which $1 \mathrm{ml}$ of each culture was boiled at $100^{\circ} \mathrm{C}$ for $8 \mathrm{~min}$. After boiling, the suspension was centrifuged for $10 \mathrm{~min}$ at $12,000 \mathrm{rpm}$. The supernatant was then used as the DNA template. Plasmid
DNA templates were extracted using a SanPrep Plasmid MiniPrep Kit (Sangon Biotech Co. Ltd.). K-serogroups of all $V$. parahaemolyticus strains were identified using commercial antisera based on agglutination tests (Denka Seiken, Tokyo, Japan) according to the manufacturer's protocol and the Chinese National Food Safety Standards: Food Microbiological Examination Vibrio parahaemolyticus Testing, GB 4789.7-2013.

\section{Primer and Probe Design}

In this study, the primers were designed as previously described (Li et al., 2019). Primers designed for development of the MLMA assay included two parts (Table 2). (1) The whole genome sequences of $418 \mathrm{~V}$. parahaemolyticus strains were sequenced in the previous study (Li et al., 2019; Bian et al., 2021) and 39 pairs of primers of different K-serogroups were designed based on specific serogroup antigen genes sequences of the 418 genomes which the bioproject numbers could be obtained from the GenBank database (https://www.ncbi.nlm.nih.gov/bioproject/PRJNA677930). Among 39 pairs of primers, the eight pairs of primers were previously reported (Li et al., 2019). (2) Additionally, 18 pairs of primers targeting 18 rare K-serogroup gene sequences were designed based on the $w z y$ or $w z x$ gene as previously described (Pang et al., 2019) using sequence information obtained from the GenBank database (https://www.ncbi.nlm.nih.gov/). The left hybridization-ligation oligonucleotide probe (L) was purified using polyacrylamide gel electrophoresis, while the right hybridization-ligation oligonucleotide probe $(\mathrm{R})$ was labeled at the $5^{\prime}$ end with the phosphate group $\left(5^{\prime} \mathrm{P}\right)$ and purified using highperformance liquid chromatography. Universal primer and fluorophore-labeled probes with the reporter fluorophores carboxy-X-rhodamine (ROX), carboxyfluorescein (FAM), or indodicarbocyanine-5 (Cy5) at the $5^{\prime}$ end and with Black Hole Quencher at the $3^{\prime}$ end were obtained as described in our previous study (Jiang et al., 2017) (Table S4). All primers and probes were synthesized by Sangon Biotech Co. Ltd.

\section{Design of the MLMA Assay}

The two key steps involved in the MLMA assay are (1) a hybridization-ligation process and (2) a PCR amplification and melt curve analysis ( $\mathrm{Li}$ et al., 2019). In step 1, the reaction is conducted in a $10 \mu \mathrm{l}$ reaction mixture containing $1.5 \mu \mathrm{l}$ of ligation probe mix (0.63-10.0 nM of each hybridization oligonucleotide probe) (Table 2), $1 \mathrm{U}$ Taq DNA ligase and $1 \mu \mathrm{l}$ DNA ligase buffer (New England Biolabs, Beijing, China), $1.5 \mu$ sterilized water, and $5 \mu \mathrm{l}$ DNA template. The reaction was performed in a T3 Thermocycler (Biometra, Germany) under the following parameters: denaturation of the initial reaction, which only contained ligation probe mix and DNA template, at $95^{\circ} \mathrm{C}$ for $5 \mathrm{~min}$, followed by a reduction to $75^{\circ} \mathrm{C}$ to pause the reaction, after which $3.5 \mu \mathrm{l}$ containing the remainder of the reaction mixture were added and the samples were incubated at $60^{\circ} \mathrm{C}$ for $60 \mathrm{~min}$, followed by $95^{\circ} \mathrm{C}$ for $5 \mathrm{~min}$. In step 2 , the reaction was conducted in a $50 \mu \mathrm{l}$ reaction mixture containing $1 \times \mathrm{PCR}$ buffer, $1.5 \mathrm{mM}$ $\mathrm{MgCl} 2,0.2 \mathrm{mM}$ deoxynucleoside triphosphate, $1 \mathrm{U}$ Taq polymerase, $0.015 \mu \mathrm{M}$ limiting primer, $0.3 \mu \mathrm{M}$ excess primer, $0.12 \mu \mathrm{M}$ fluorogenic probes, and $5 \mu \mathrm{l}$ of ligation product from step 1. The reaction was performed in a BioRad CFX96 real-time PCR 


\begin{tabular}{|c|c|c|c|c|c|}
\hline Serogroup ${ }^{a}$ & Gene loci & Tube & $\begin{array}{l}\text { Fluorescence } \\
\text { channel }\left(\mathrm{Tm} /{ }^{\circ}\right)\end{array}$ & $\begin{array}{c}\text { Sequence } \\
\left(5^{\prime}-3^{\prime}\right)\end{array}$ & Hybridization-ligation oligonucleotide probe sequence $\left(5^{\prime} \rightarrow 3^{\prime}\right)^{\mathbf{b}}$ \\
\hline K3 & VP24500037 & 1 & $(\mathrm{FAM}, 74.5)$ & F & GTGGCAGGGCGCTACGAACAATCCTATCGGTCCTTCATCGCTCAGCCTTCACCGGTTCTTGCTGACGATCTATGTGTTAACGTAG \\
\hline K5 & VP19500014 & 1 & $($ Cy5,61.0) & $\mathrm{F}$ & GTGGCAGGGCGCTACGAACAATCCTACGGTGAGGACCTTTGAGATTGGCATCACCCCGGAAGGCTGTCGCTCTACTTCTATATCG \\
\hline & & & & $\mathrm{R}$ & AGATGAGCCAACATTATTAGTCGGGTTGAGATTGGATCTTGCTGGGC \\
\hline K6 & VP0223 & 1 & $(\mathrm{ROX}, 74.5)$ & $\begin{array}{l}F \\
R\end{array}$ & $\begin{array}{l}\text { GTGGCAGGGCGCTACGAACAATCCTAACGACTCTGGCTGCTCGTTCGTGACGCCGTTAGAACCTAAGTCTAATTATGCAGTCA } \\
\text { CTGGGCTATATITCTATGACAGTCGCGTAATAGTGAGATTGGATCTTGCTGGGC }\end{array}$ \\
\hline K8 & VPBB0234 & 1 & $(\mathrm{ROX}, 70.0)$ & $\begin{array}{l}\mathrm{F} \\
\mathrm{R}\end{array}$ & $\begin{array}{l}\text { GTGGCAGGGCGCTACGAACAATCCTAACGACTCTAGCTGCTCGTTCGTGACGGAACTTGATTGAAGCAAGGGAACATTCTT } \\
\text { CGGTGAGTATGATTAATACATTGTCACTTCTGAGATTGGATCTTGCTGGGC }\end{array}$ \\
\hline K9 & VP13500017 & 1 & $($ FAM,66.0) & $\begin{array}{l}\mathrm{F} \\
\mathrm{R}\end{array}$ & $\begin{array}{l}\text { GTGGCAGGGCGCTACGAACAATCCTATCGGTCCTTATCGCTCACCCTTCACCGGCGGAGTGATTATAAGGAGGAGTGCTATAATG } \\
\text { TGGGTCGGGAATCGGTGTCAGTGTAATGAGATTGGATCTTGCTGGGC }\end{array}$ \\
\hline K11 & VP10700010 & 1 & $($ Cy $5,70.5)$ & $\begin{array}{l}\mathrm{F} \\
\mathrm{R}\end{array}$ & $\begin{array}{l}\text { GTGGCAGGGCGCTACGAACAATCCTACGGTGAAGCCCTTGGCAGGTCGGTATCACCCGGTTCGGTTAAGATAAGTACTTGGGTAGAT } \\
\text { GTTCATTGCACTCGCTCTGCTTATTACGTGAGATTGGATCTTGCTGGGC }\end{array}$ \\
\hline K12 & VP17900014 & 1 & $($ FAM,51.0) & $\begin{array}{l}\mathrm{F} \\
\mathrm{R}\end{array}$ & $\begin{array}{l}\text { GTGGCAGGGCGCTACGAACAATCCTATCGCTCCTTCATAGCTCAGACTTCATCGGGAACTAGTAAGTTIATATAACCCGGCATTGC } \\
\text { ATTGTATTGAAGAGCAACGTGATTITCCTCAATCGTGAGATTGGATCTTGCTGGGC }\end{array}$ \\
\hline K13 & VP9800011 & 1 & $(\mathrm{FAM}, 61.5)$ & $\begin{array}{l}F \\
R\end{array}$ & $\begin{array}{l}\text { GTGGCAGGGCGCTACGAACAATCCTATCGGTCCTTCATGGCTCAGTCTTCACCGGGACCTCTTGGATGGGATTCTAATATACACG } \\
\text { ATAAGTTGATAAAGTATTCACTTGGAGACTCTGAGATTGGATCTTGCTGGGC }\end{array}$ \\
\hline K17 & VP4300018 & 1 & $(\mathrm{ROX}, 57.5)$ & $\begin{array}{l}\mathrm{F} \\
\mathrm{R}\end{array}$ & $\begin{array}{l}\text { GTGGCAGGGCGCTACGAACAATCCTAACGACTCTAGCTTCTCGTTAGTGACGGCTTCTTGACCACACGTTATTGTACCAAT } \\
\text { CTTGCTAGGTACAAAGCCAAAAGCAGCCATGAGATTGGATCTTGCTGGGC }\end{array}$ \\
\hline K18 & VP19700016 & 1 & (Су5,53.5) & $\begin{array}{l}\mathrm{F} \\
\mathrm{R}\end{array}$ & $\begin{array}{l}\text { GTGGCAGGGCGCTACGAACAATCCTACGGTGAAGCCATTGCCAGGTGGTATACCCGAGTTGGCATTGATGCTCATCTCTCCT } \\
\text { ATCTATTCTGTTATAAAATTGATGTCTACGGACGTGAGATTGGATCTTGCTGGGC }\end{array}$ \\
\hline K25 & VP13200012 & 1 & $(\mathrm{ROX}, 66.5)$ & $\mathrm{F}$ & $\begin{array}{l}\text { GTGGCAGGGCGCTACGAACAATCCTAACGACTCTGTCTTCTCGTTCGTGACGGCTTATCTAGTCGTTCTTCATTGGTGAGAAAG } \\
\text { CTITCAACTCCAAAAGTATCGTGATAAAATGAGATTGGATCTTGCTGGGC }\end{array}$ \\
\hline K28 & VP5300035 & 1 & (FAM,57.0) & $\begin{array}{l}\mathrm{F} \\
\mathrm{R}\end{array}$ & $\begin{array}{l}\text { GTGGCAGGGCGCTACGAACAATCCTATCCGTCTTATCGCTCAGCCTTCATCGGCCATATTTGACCCTTCAGTTAGGTATCG } \\
\text { TTGTTCAATCTTGCTGATGAGCTAAATGAGCGATGAGATTGGATCTTGCTGGGC }\end{array}$ \\
\hline K29 & VP24700016 & 1 & $(\mathrm{ROX}, 63.5)$ & $\begin{array}{l}F \\
R\end{array}$ & $\begin{array}{l}\text { GTGGCAGGGCGCTACGAACAATCCTAACGACTCTAGCTGCTTGTTCGTGACGTGATAAGTATTCTTGATATCGAAAGTGGCGA } \\
\text { GTGTTIACAATAAGAAGATTAAAATTGAGAGTGAGATTGGATCTTGCTGGGC }\end{array}$ \\
\hline K36 & VP12200010 & 1 & $(\mathrm{FAM}, 70.0)$ & $\begin{array}{l}\mathrm{F} \\
\mathrm{R}\end{array}$ & $\begin{array}{l}\text { GTGGCAGGGCGCTACGAACAATCCTATCGGTCCTTCATCGCTCGGCCTTCACCGGATTGGCAAAAAAGGTTTGTTCAATCGAAT } \\
\text { CTAGCAATKTGGCTCTAAGTRGTTGAATGTAGGTGAGATTGGATCTTGCTGGGC }\end{array}$ \\
\hline K41 & VP23400016 & 1 & (Сy5,74.0) & $\begin{array}{l}\mathrm{F} \\
\mathrm{R}\end{array}$ & $\begin{array}{l}\text { GTGGCAGGGCGCTACGAACAATCCTACGGTGAGGCCCTTGGCAGGTTGCTATCACCCAAACAAAGCTCTCAAGGATGCTAAGCTT } \\
\text { GATGGTGCGAGTATIITATATATCGGGTGGATGAGATTGGATCTTGCTGGGC }\end{array}$ \\
\hline K42 & VP400015 & 1 & (Сy5,56.0) & $\begin{array}{l}F \\
R\end{array}$ & $\begin{array}{l}\text { GTGGCAGGGCGCTACGAACAATCCTACGGTGCGGACCTTGCCGATTGGCATCACCCTGTAACTCTAAACCTAAGTCTTCATGGCTGA } \\
\text { TGTITGTITGCGGTATGTCACAAACTCAGTGAGATTGGATCTTGCTGGGC }\end{array}$ \\
\hline K44 & VP11300018 & 1 & (Су5,58.5) & $\begin{array}{l}F \\
R\end{array}$ & $\begin{array}{l}\text { GTGGCAGGGCGCTACGAACAATCCTACGGTGAGGACCTTGCCGATTGGCATCACCCGCTITGCAATGATAAGTAATGCTCAGGTAA } \\
\text { TGTATCTTCTCTACAAGAAAATCCCTTGCTTGGATGAGATTGGATCTTGCTGGGC }\end{array}$ \\
\hline K56 & VP33400015 & 1 & $(\mathrm{ROX}, 60.0)$ & $\begin{array}{l}F \\
R\end{array}$ & $\begin{array}{l}\text { GTGGCAGGGCGCTACGAACAATCCTAACGACACTGGCTGCTGGTCCGTGACGTAGAACACTCAAACCGGAAGTTCATCGCAA } \\
\text { GAATGGATGCTGATGATATTCAGAGCCATGAGATTGGATCTTGCTGGGC }\end{array}$ \\
\hline K60 & VP1600020 & 1 & (Cy5,66.0) & $\begin{array}{l}F \\
R\end{array}$ & $\begin{array}{l}\text { GTGGCAGGGCGCTACGAACAATCCTACGGTGAAGCCCTTCGCAGGTCGGTATCACCCCTTCTTGTACAGCCTTAAGAGCGGGA } \\
\text { TTGGTGCCATITCATCCGGATTGGTGTGAGATTGGATCTTGCTGGGC }\end{array}$ \\
\hline K68 & VP16100014 & 1 & $(\mathrm{ROX}, 54.0)$ & $\begin{array}{l}F \\
R\end{array}$ & $\begin{array}{l}\text { GTGGCAGGGCGCTACGAACAATCCTAACGACTATGGCTTCTCGTTGGTGACGCGATACTAATGACTCAGATGTATGCCCAGGATT } \\
\text { TIAACAGAATGTGGGGCCAAGAAAGTATGAGATTGGATCTTGCTGGGC }\end{array}$ \\
\hline K70 & wzy & 1 & $(\mathrm{ROX}, 50.5)$ & $\begin{array}{l}\mathrm{F} \\
\mathrm{R}\end{array}$ & $\begin{array}{l}\text { GTGGCAGGGCGCTACGAACAATCCTAACGACTCTATCTGCTTGTTAGTGACGATACACAATCGTTCAACATAATAAGGCAAGC } \\
\text { ACTAGGGATGGCAATTGTCTTATTCGCTGGACTGAGATTGGATCTTGCTGGGC }\end{array}$ \\
\hline K1 & VP30500018 & 2 & $(\mathrm{ROX}, 51.0)$ & $\begin{array}{l}F \\
R\end{array}$ & $\begin{array}{l}\text { GTGGCAGGGCGCTACGAACAATCCTAACGACTCTATCTGCTTGTTAGTGACGATGGGATTAGCTATACCTAGGCTAGCCGGT } \\
\text { GCTCATGGAGACCCTAACTATAACTCAGTTGAGATTGGATCTTGCTGGGC }\end{array}$ \\
\hline K4 & VP31900014 & 2 & $(\mathrm{ROX}, 54.0)$ & $\begin{array}{l}F \\
R\end{array}$ & $\begin{array}{l}\text { GTGGCAGGGCGCTACGAACAATCCTAACGACTATGGCTTCTCGTTGGTGACGTTGGTATTGGTGTTGACTACGGATATGGT } \\
\text { GCTCCAAGAATGCAAGGTITCTGAGTGAACTGAGATTGGATCTTGCTGGGC }\end{array}$ \\
\hline
\end{tabular}




\begin{tabular}{|c|c|c|c|c|c|}
\hline Serogroup $^{a}$ & Gene loci & Tube & $\begin{array}{l}\text { Fluorescence } \\
\text { channel }\left(\mathrm{Tm} /{ }^{\circ} \mathrm{C}\right)\end{array}$ & $\begin{array}{l}\text { Sequence } \\
\left(5^{\prime}-3^{\prime}\right)\end{array}$ & Hybridization-ligation oligonucleotide probe sequence $\left(5^{\prime} \rightarrow 3^{\prime}\right)^{b}$ \\
\hline K19 & VP19200012 & 2 & $(\mathrm{ROX}, 57.0)$ & $\begin{array}{l}\mathrm{F} \\
\mathrm{R}\end{array}$ & $\begin{array}{l}\text { GTGGCAGGGCGCTACGAACAATCCTAACGACTCTAGCTTCTCGTTAGTGACGGATATGCAAGACTTGCAAAAGCTCATCACAA } \\
\text { AATCATCTAGGTTGATGTGGGCTCTITGTTGAGATTGGATCTTGCTGGGC }\end{array}$ \\
\hline K20 & VP20600013 & 2 & $(\mathrm{ROX}, 59.0)$ & $\begin{array}{l}\mathrm{F} \\
\mathrm{R}\end{array}$ & $\begin{array}{l}\text { GTGGCAGGGCGCTACGAACAATCCTAACGACACTGGCTGCTGGTCCGTGACGGCACTGAATACGCCTTAAAAACTCTAATAGC } \\
\text { TTGGGCTCAGTTGTTGCAACTATTGGTGTTGAGATTGGATCTTGCTGGGC }\end{array}$ \\
\hline K21 & VP43900016 & 2 & (Сy5,61.5) & $\begin{array}{l}\mathrm{F} \\
\mathrm{R}\end{array}$ & $\begin{array}{l}\text { GTGGCAGGGCGCTACGAACAATCCTACGGTGAGGACCTTGCAGATTGGCATCACCCGTTAGCTGTGGAAGACGTGTATTGTTGAA } \\
\text { AACTGTAACAACGACATAATGATACTATTCGATGTGAGATTGGATCTTGCTGGGC }\end{array}$ \\
\hline K23 & VP20200014 & 2 & $(\mathrm{FAM}, 74.0)$ & $\begin{array}{l}\mathrm{F} \\
\mathrm{R}\end{array}$ & $\begin{array}{l}\text { GTGGCAGGGCGCTACGAACAATCCTATCGGTCCTTCATCGCTCAGCCTTCACCGGCGAAATTCATAGCGATCTTGAGACTTCAA } \\
\text { TCTTGGTTGTAGGAATTATGCCATATACCATGAGATTGGATCTTGCTGGGC }\end{array}$ \\
\hline K30 & VP19000009 & 2 & (Cy5,58.5) & $\begin{array}{l}\mathrm{F} \\
\mathrm{R}\end{array}$ & $\begin{array}{l}\text { GTGGCAGGGCGCTACGAACAATCCTACGGTGAGGACCTTTGCCGATTGGCATCACCCATTCAAGTCATGGATTACTGGTCCTTGTATT } \\
\text { TTGATCGGTGCTGGTTGGTGGCAGTATGGTGAGATTGGATCTTGCTGGGC }\end{array}$ \\
\hline K31 & VP20500017 & 2 & $($ FAM,69.5) & $\begin{array}{l}\mathrm{F} \\
\mathrm{R}\end{array}$ & $\begin{array}{l}\text { GTGGCAGGGCGCTACGAACAATCCTATCGGTCCTTCATCGCTCGGCCTTCACCGGTTGGGTATGCTTCCGTCATTTAGAACTATT } \\
\text { CACTACGGAGATACAGCCAACTACTATGACTGAGATTGGATCTTGCTGGGC }\end{array}$ \\
\hline K32 & VP9900015 & 2 & $($ FAM,65.5) & $\begin{array}{l}\mathrm{F} \\
\mathrm{R}\end{array}$ & $\begin{array}{l}\text { GTGGCAGGGCGCTACGAACAATCCTATCGGTCCTIATCGCTCACCCTTCACCGGCCAATCGATGAACCAAATTAGGCAATTTGC } \\
\text { TGCAATCGCTGTGTCACTITIGCCTTGCTTGAGATTGGATCTTGCTGGGC }\end{array}$ \\
\hline K33 & VP20100010 & 2 & $($ FAM,61.0) & $\begin{array}{l}\mathrm{F} \\
\mathrm{R}\end{array}$ & $\begin{array}{l}\text { GTGGCAGGGCGCTACGAACAATCCTATCGGTCCTTCATGGCTCAGTCTTCACCGGGGTATTGGATTGCAGTTAGATGCGGAGTAA } \\
\text { CGAAGCTTCATATCACCGGGATGCAAAGATGAGATTGGATCTTGCTGGGC }\end{array}$ \\
\hline K34 & VP1500017 & 2 & $(\mathrm{ROX}, 70.0)$ & $\begin{array}{l}\mathrm{F} \\
\mathrm{R}\end{array}$ & $\begin{array}{l}\text { GTGGCAGGGCGCTACGAACAATCCTAACGACTCTAGCTGCTCGTTCGTGACGCTCACAATTGTAAGACGATTACTTACAGCA } \\
\text { CGGATTGTATGTTGATTACTCATTAAGTGAAATGAGATTGGATCTTGCTGGGC }\end{array}$ \\
\hline K37 & VP23900011 & 2 & $($ FAM,57.0) & $\begin{array}{l}\mathrm{F} \\
\mathrm{R}\end{array}$ & $\begin{array}{l}\text { GTGGCAGGGCGCTACGAACAATCCTATCCGTCTTATCGCTCAGCCTTCATCGGAGATTCTGACCTCTATGGGAAAGGGTAT } \\
\text { GGGTAGGGTGGTCCTATTAGTGATITGAGATTGGATCTTGCTGGGC }\end{array}$ \\
\hline K38 & VP20000015 & 2 & $($ FAM,52.0) & $\begin{array}{l}\mathrm{F} \\
\mathrm{R}\end{array}$ & $\begin{array}{l}\text { GTGGCAGGGCGCTACGAACAATCCTATCGCTCCTTCATAGCTCAGACTTCATCGGGGTATCGCCGTTACTGGCACATACCATG } \\
\text { ATTATTGTCCGCTATCTGTTGACTTGGGATTGAGATTGGATCTTGCTGGGC }\end{array}$ \\
\hline K48 & VP22900017 & 2 & (Су5,55.5) & $\begin{array}{l}\mathrm{F} \\
\mathrm{R}\end{array}$ & $\begin{array}{l}\text { GTGGCAGGGCGCTACGAACAATCCTACGGTGCGGACCTTGCCGATTGGCATCACCCCCAGTTCGTCTTGAAACGTATTTGCCAAA } \\
\text { CGCTCTTGTAGGCTTCCTCTCATAATTGAGATTGGATCTTGCTGGGC }\end{array}$ \\
\hline K49 & VP23800014 & 2 & (Сy5,50.5) & $\begin{array}{l}\mathrm{F} \\
\mathrm{R}\end{array}$ & $\begin{array}{l}\text { GTGGCAGGGCGCTACGAACAATCCTACGGTGAAGCCATTGCCAGGTGGTATACCTGTTTGATTATAACCCTTATGGTGCGATTCT } \\
\text { GGGGTAGGTTCAATGGGGTTGTATGAATGAGATTGGATCTTGCTGGGC }\end{array}$ \\
\hline K55 & VP100017 & 2 & $(\mathrm{ROX}, 62.5)$ & $\begin{array}{l}\mathrm{F} \\
\mathrm{R}\end{array}$ & $\begin{array}{l}\text { GTGGCAGGGCGCTACGAACAATCCTAACGACTCTAGCTGCTTGTTCGTGACGGCTAATTCTCAATCAAATGGATGGGACTGG } \\
\text { TATTCATACAAAGATTCGTTCGAGCAAATTCTTGAGATTGGATCTTGCTGGGC }\end{array}$ \\
\hline K63 & VP3400018 & 2 & $(\mathrm{ROX}, 66.5)$ & $\begin{array}{l}\mathrm{F} \\
\mathrm{R}\end{array}$ & $\begin{array}{l}\text { GTGGCAGGGCGCTACGAACAATCCTAACGACTCTGTCTTCTCGTTCGTGACGCGCTAAAGGGTGGGGAGATAACGGGATA } \\
\text { GGAATACATCAAACTGGTGGGCTAGATAATCGTGAGATTGGATCTTGCTGGGC }\end{array}$ \\
\hline K65 & wzy & 2 & $(\mathrm{ROX}, 73.5)$ & $\begin{array}{l}\mathrm{F} \\
\mathrm{R}\end{array}$ & $\begin{array}{l}\text { GTGGCAGGGCGCTACGAACAATCCTAACGACTCTGGCTGCTCGTTCGTGACGAACAAAGATTCCTTCCGGAGATAAATATTCC } \\
\text { ATTCCTGGTATATCTGGCGTTCGGCTGTATGAGATTGGATCTTGCTGGGC }\end{array}$ \\
\hline K67 & wzy & 2 & (Cy5,66.0) & $\begin{array}{l}\mathrm{F} \\
\mathrm{R}\end{array}$ & $\begin{array}{l}\text { GTGGCAGGGCGCTACGAACAATCCTACGGTGAAGCCCTCGCAGGTCGGTATCACCCTATGGACGCAGTCGCGCAATGATGTITGCA } \\
\text { CTGCAATATATTATTATGACAGCTTCCGTTCTCTGAGATTGGATCTTGCTGGGC }\end{array}$ \\
\hline K69 & VP3300015 & 2 & (Cy5,70.5) & $\begin{array}{l}\mathrm{F} \\
\mathrm{R}\end{array}$ & $\begin{array}{l}\text { GTGGCAGGGCGCTACGAACAATCCTACGGTGAAGCCCTTGGCAGGTCGGTATCACCCGTAGTCCTTGCATACCCCGTGTTAATAG } \\
\text { TATCTGCCGGATTGCTCAGGAATGGACAATGAGATTGGATCTTGCTGGGC }\end{array}$ \\
\hline K71 & VP3200012 & 2 & (Cy5,74.5) & $\begin{array}{l}\mathrm{F} \\
\mathrm{R}\end{array}$ & $\begin{array}{l}\text { GTGGCAGGGCGCTACGAACAATCCTACGGTGAGGCCCTTGGCAGGTGGTATCACCCGGAATTGAAGTATGTTCTTGAAGAGGGA } \\
\text { CCATGCTITATCGTGCAGGACAAACTAAGTGAGATTGGATCTTGCTGGGC }\end{array}$ \\
\hline K7 & wzy & 3 & $(\mathrm{ROX}, 74.0)$ & $\begin{array}{l}F \\
R\end{array}$ & $\begin{array}{l}\text { GTGGCAGGGCGCTACGAACAATCCTAACGACTCTGGCTGCTCGTTCGTGACGATCTTATCGAGTCAACAGCTATGTATGCGA } \\
\text { GACTTAGTTACTTCTACTATGGCGATGGTTGGAGTGAGATTGGATCTTGCTGGGC }\end{array}$ \\
\hline K15 & $w z y$ & 3 & $(\mathrm{ROX}, 57.0)$ & $\begin{array}{l}\mathrm{F} \\
\mathrm{R}\end{array}$ & $\begin{array}{l}\text { GTGGCAGGGCGCTACGAACAATCCTAACGACTCTAGCTTCTCGTTAGTGACGACAGGACATACCCAAAAGACATTGGCA } \\
\text { CTGGCTGGACTGGAACCCAGTTTTCATGTGAGATTGGATCTTGCTGGGC }\end{array}$ \\
\hline K22 & $w z x$ & 3 & $(\mathrm{ROX}, 54.0)$ & $\begin{array}{l}\mathrm{F} \\
\mathrm{R}\end{array}$ & $\begin{array}{l}\text { GTGGCAGGGCGCTACGAACAATCCTAACGACTATGGCTTCTCGTTGGTGACGTTTCGACAATCCAATTTCTGAGCGAGTCTTA } \\
\text { CCTTCATTATGCGTTACAAATCCATCTACTGAGATTGGATCTTGCTGGGC }\end{array}$ \\
\hline K24 & wzy & 3 & $(\mathrm{ROX}, 70.0)$ & $\begin{array}{l}\mathrm{F} \\
\mathrm{R}\end{array}$ & $\begin{array}{l}\text { GTGGCAGGGCGCTACGAACAATCCTAACGACTCTAGCTGCTCGTTCGTGACGCTTACACGCAATTITAGAGGCGTTACAATAT } \\
\text { CTGCTATTGGTATTGGGATTGACTATCTCTAGCTGAGATTGGATCTTGCTGGGC }\end{array}$ \\
\hline
\end{tabular}


Serogroup $^{\mathrm{a}}$ Gene loci Tube

Fluorescence

channel $\left(\mathrm{Tm} /{ }^{\circ} \mathrm{C}\right)$

Sequence

$\left(5^{\prime}-3^{\prime}\right)$

Hybridization-ligation oligonucleotide probe sequence $\left(5^{\prime} \rightarrow 3^{\prime}\right)^{b}$

$\begin{array}{llll}\text { K39 } & \text { wzy } & 3 & (\mathrm{ROX}, 50.5)\end{array}$

(ROX,62.5)

(ROX,66.5)

K45
GTGGCAGGGCGCTACGAACAATCCTAACGACTCTATCTGCTTGTTAGTGACGGTGGTAGGTTCTIGCATACATACTGCTCCTG AACGATTCTGTGTITCGTACAACTIATATGGTGTATGAGATTGGATCTTGCTGGGC

AACGATTCTGTGTTCGTACAACTIATATGGTGTATGAGATTGGATCTTGCTGGGC TTGGGAGATGGGGTTTCAAGCATTATTTAGAGTTTGAGATTGGATCTTGCTGGGC

GTGGCAGGGCGCTACGAACAATCCTAACGACTCTGTCTTCTCGTTCGTGACGTTTGCGTTGTCTCTATTGTATTACTTGGG CTATATITCCTAGCTAAGGAAATGGGGCAGATAAGTGAGATTGGATCTTGCTGGGC

GTGGCAGGGCGCTACGAACAATCCTAACGACACTGGCTGCTGGTCCGTGACGAATACTCTATGGGTGGCGATATATTGCGATA GGCTATGGCGGGATTGATGCAAGTGCTTATAAGTGAGATTGGATCTTGCTGGGC

GTGGCAGGGCGCTACGAACAATCCTACGGTGAGGACCTTTGCCGATTGGCATCACCCTGCAGCACTCTCTTGATTACAGTGATGATTA CGGTCTCGCATCAATTCTGGCTACTITGCTGCTGAGATTGGATCTTGCTGGGC

GTGGCAGGGCGCTACGAACAATCCTACGGTGAGGACCTTGCAGATTGGCATCACCCATGCATTAAAGGAGTACAACCACTCCA CAGGCTCTGGCCTCGCTTATTATITTAGGTGAGATTGGATCTTGCTGGGC

GTGGCAGGGCGCTACGAACAATCCTACGGTGAAGCCCTTCGCAGGTCGGTATCACCCAGCTATCTGATGACCTTGTGGCATTAAA GAAATGGTTTCTAAAGTAGTTCCTATTGTCGCCTTTGAGATTGGATCTTGCTGGGC

GTGGCAGGGCGCTACGAACAATCCTACGGTGAGGCCCTTGGCAGGTTGCTATCACCCATGGTGAATTCTGGGTAAGAAACAACCG TACGAGTCTTGGGGGCAATATGTATITCAGCTCTGAGATTGGATCTTGCTGGGC

GTGGCAGGGCGCTACGAACAATCCTACGGTGAAGCCATTGCCAGGTGGTATACCCTGTCAGACTCCGGAATTCCCGCTTATCAT

CTATAAAGACTITGCTCTACGATTCAAGTAATTCGTGAGATTGGATCTTGCTGGGC

GTGGCAGGGCGCTACGAACAATCCTACGGTGCGGACCTITGCCGATTGGCATCACCCACGACGAGTGTTCAATTGGATGACGATAGTTC AATGTTATCTCTCTACTAAACGGAGAGTTAGGAGTGAGATTGGATCTTGCTGGGC

GTGGCAGGGCGCTACGAACAATCCTACGGTGAAGCCCTTGGCAGGTCGGTATCACCCAAGCCTTCAGCCATCCCCAAGAATCCA CCTGTTCTTGGAGAGTTAAGACCATCAATACTTGAGATTGGATCTTGCTGGGC

GTGGCAGGGCGCTACGAACAATCCTATCGGTCCTTATCGCTCACCCTTCACCGGGATCGCATGACTCAGTCATCGTGAAA GAAAGGCACAACTTTGTAGAGATTTCTGTTGAGATTGGATCTTGCTGGGC

GTGGCAGGGCGCTACGAACAATCCTATCGGTCCTTCATCGCTCGGCCTTCACCGGAACCAGAAGCGCCAGTAGTACCTGAAAAAGCA CCTGTGGCTTCTGCTGTGAATCCTTGGATTTGAGATTGGATCTTGCTGGGC

${ }^{2} K 3, K 6, K 8, K 9, K 25, K 29, K 56$ and $K 68$ were the 8 previously reported $K$-serogroups; $K 7, K 15, K 22, K 24, K 39, K 40, K 43, K 45, K 46, K 51, K 52, K 53, K 54, K 59, K 64, K 65, K 67$ and $K 70$ were the 18 rare $K$-serogroups.

${ }^{b}$ Bold typeface indicates universal primer sequences used during the LATE-PCR amplification step; whereas fluorescent detection probe sequences are underlined. 
system (BioRad Inc., Hercules, CA, USA) under the following conditions: host start at $95^{\circ} \mathrm{C}$ for $3 \mathrm{~min}$, then 38 cycles of $95^{\circ} \mathrm{C}$ for $10 \mathrm{~s}, 57^{\circ} \mathrm{C}$ for $20 \mathrm{~s}$, and $72^{\circ} \mathrm{C}$ for $20 \mathrm{~s}$, followed by $95^{\circ} \mathrm{C}$ for $1 \mathrm{~min}$, $40^{\circ} \mathrm{C}$ for $2 \mathrm{~min}$, and then an increase to $85^{\circ} \mathrm{C}$ in $1^{\circ} \mathrm{C}$ steps with $5 \mathrm{~s}$ between each step. All fluorescent signal intensity was captured by ROX, FAM, and Cy5 channels. The melting temperature (Tm) values of melt curve analysis were obtained automatically using CFX Manager 3.0 software.

\section{Analytical Performance of the MLMA Assay}

To determine the limit of detection (LOD) of the assay, a series of 10 -fold dilutions in triplicates of purified DNA from $10.0 \mathrm{ng} / \mu \mathrm{l}$ to $0.01 \mathrm{ng} / \mu \mathrm{l}$ were analyzed. To evaluate the intra-assay and interassay reproducibility, two sets of 10 -fold dilutions in triplicate from $10.0 \mathrm{ng} / \mu \mathrm{l}$ to $1.0 \mathrm{ng} / \mu \mathrm{l}$ were analyzed and the standard deviations and coefficient of variation values were calculated. Each concentration of the assay was analyzed in triplicate.

\section{Evaluation of the MLMA Assay Using a Double-Blind Test}

The 595 strains were selected to evaluate the specificity and sensitivity of the MLMA assay. The K-serogroups of the 595 isolates were distinguished based on the MLMA assay method compared with the agglutination tests (Denka Seiken) using commercial antisera according to the manufacturer's instructions with a double-blind test. The genomic DNA was extracted from the 595 isolates using the boiled lysates protocol. Procedures were performed according to the protocols described above. Evaluation of consistency between the MLMA assay and traditional serological methods was performed using Kappa analysis.

\section{Statistical Analysis}

The SPSS 23.0 statistical software package was used for statistical analysis. The positive detection rate of the MLMA assay and traditional serological method was compared with the paired $\chi^{2}$ test, and a $\mathrm{P}<0.01$ was considered to indicate statistical significance.

\section{Ethics}

$V$. parahaemolyticus isolates from the Shenzhen Center for Disease Control and Prevention were de-identified and anonymized to protect patient privacy and confidentiality. Therefore, ethical clearance was not required.

\section{RESULTS}

\section{Identification of $57 \mathrm{~V}$. parahaemolyticus K-Serogroups}

A three-tube system was set up with three fluorescence channels (ROX, FAM, and Cy5) in each tube. In the first tube, the ROX channel detected the K-serogroups K70, K68, K17, K56, K29, $\mathrm{K} 25, \mathrm{~K} 8$, and $\mathrm{K} 6$, the Cy5 channel detected the K-serogroups $\mathrm{K} 18, \mathrm{~K} 42, \mathrm{~K} 44, \mathrm{~K} 5, \mathrm{~K} 60, \mathrm{~K} 11$, and $\mathrm{K} 41$ and the FAM channel detected the K-serogroups K12, K28, K13, K9, K36, and K3. In the second tube, the ROX channel detected the K-serogroups K1,
$\mathrm{K} 4$, K19, K20, K55, K63, K34, and K65, the Cy5 channel detected K49, K48, K30, K21, K67, K69, and K71 and the FAM channel detected the K-serogroups K38, K37, K33, K32, K31, and K23. In the third tube, the ROX channel detected the K-serogroups K39, $\mathrm{K} 22$, K15, K45, K40, K43, K24, and K7, the Cy5 channel detected the K-serogroups K54, K59, K46, K51, K52, K64, and K53 and the FAM channel detected the SUC2 gene as an internal control (IC) and the toxR gene as a confirmation of $V$. parahaemolyticus. The combined use of fluorescence channels and designed Tm values for targeting specific gene loci of K-serogroups in each tube are listed in Table 3.

\section{Performance Characteristics of the MLMA Assay}

With the use of 59 pairs of ligation oligonucleotides in a threetube system, the MLMA assay was able to detect all target genes of $57 \mathrm{~K}$-serogroups and one IC and the toxR gene, which yielded expected Tm values that represented distinguished K-serogroups (Figure 1). The specificity study showed no cross-reactivity among the $57 \mathrm{~K}$-serogroups. The limit of detection (LOD) of the assay for all target genes of K-serogroups ranged from 0.1 to $1.0 \mathrm{ng} / \mu \mathrm{l}$ at the DNA level. The intra-assay and inter-assay reproducibility study demonstrated that the measurement of Tm values has highly reproducible traits. Specifically, the largest SD value of the mean Tm was no more than $1^{\circ} \mathrm{C}$ and the $\mathrm{CV}$ value ranged from 0 to $1 \%$ (Table 4 ).

\section{Evaluation of the MLMA Assay}

Of the 595 isolates, 377 were identified and classified to $29 \mathrm{~K}$ serogroups, and the remaining 218 isolates were untypeable by using the MLMA assay. Among 377 isolates, the 18 could be serotyped using the MLMA assay, but could not be serotyped using the conventional serological method. Sanger sequencing of the 18 isolates demonstrated $100 \%$ concordance with the MLMA assay (Table S5). Evaluation using the McNemar test revealed that the difference was statistically significant $(\mathrm{P}=0.00)$, the positive detection rate of MLMA (63.36\%) was higher than that of conventional serotyping (60.34\%), and the MLMA assay sensitivity and specificity were $100 \%$ and $92.37 \%$, respectively. Moreover, the MLMA assay showed $96.97 \%$ consistency with the conventional serological method for all isolates. The kappa value between the MLMA assay and the traditional serological method was 0.936 (Table 5). The results of identification using the MLMA assay and the traditional serological method are listed in Table S6. In addition, five rare K-serogroups, K24, K52, K54, K67, and K70, were identified using the established MLMA assay (Table S7).

\section{DISCUSSION}

$V$. parahaemolyticus is the leading causal agent of human acute gastroenteritis and has become a global public health issue (Letchumanan et al., 2014; Han et al., 2019). $V$. parahaemolyticus serotyping is considered to be the initial step for timely detection and source tracking of vibriosis 
TABLE 3 | Identification matrix of gene targets in a three-tube multiplex ligation reaction based on probe melting curve analysis (MLMA) system.

\begin{tabular}{|c|c|c|c|c|c|c|c|c|}
\hline First tube & Antigen & $\mathrm{Tm}\left({ }^{\circ} \mathrm{C}\right)$ & Second tube & Antigen & $\operatorname{Tm}\left({ }^{\circ} \mathrm{C}\right)$ & Third tube & Antigen & $\operatorname{Tm}\left({ }^{\circ} \mathrm{C}\right)$ \\
\hline \multirow[t]{8}{*}{ ROX channel } & K70 & 50.5 & ROX channel & $\mathrm{K} 1$ & 51.0 & ROX channel & K39 & 50.5 \\
\hline & K68 & 54.0 & & K4 & 54.0 & & K22 & 54.0 \\
\hline & K17 & 57.5 & & K19 & 57.0 & & K15 & 57.0 \\
\hline & K56 & 60.0 & & K20 & 59.0 & & K45 & 59.0 \\
\hline & K29 & 63.5 & & K55 & 62.5 & & $\mathrm{~K} 40$ & 62.5 \\
\hline & K25 & 66.5 & & K63 & 66.5 & & K43 & 66.5 \\
\hline & K8 & 70.0 & & K34 & 70.0 & & K24 & 70.0 \\
\hline & K6 & 74.5 & & K65 & 73.5 & & $\mathrm{~K} 7$ & 74.0 \\
\hline \multirow[t]{7}{*}{ Cy5 channel } & K18 & 53.5 & Cy5 channel & K49 & 50.5 & Cy5 channel & K54 & 53.0 \\
\hline & K42 & 56.0 & & K48 & 55.5 & & K59 & 56.0 \\
\hline & K44 & 58.5 & & K30 & 58.5 & & K46 & 58.5 \\
\hline & K5 & 61.0 & & K21 & 61.5 & & K51 & 61.5 \\
\hline & K60 & 66.0 & & K67 & 66.0 & & K52 & 66.0 \\
\hline & K11 & 70.5 & & K69 & 70.5 & & K64 & 70.5 \\
\hline & K41 & 74.0 & & K71 & 74.5 & & K53 & 74.0 \\
\hline \multirow[t]{6}{*}{ FAM channel } & K12 & 51.0 & FAM channel & K38 & 52.0 & FAM channel & IC & 65.0 \\
\hline & K28 & 57.0 & & K37 & 57.0 & & VP & 70.0 \\
\hline & K13 & 61.5 & & K33 & 61.0 & & & \\
\hline & K9 & 66.0 & & K32 & 65.5 & & & \\
\hline & K36 & 70.0 & & K31 & 69.5 & & & \\
\hline & K3 & 74.5 & & K23 & 74.0 & & & \\
\hline
\end{tabular}
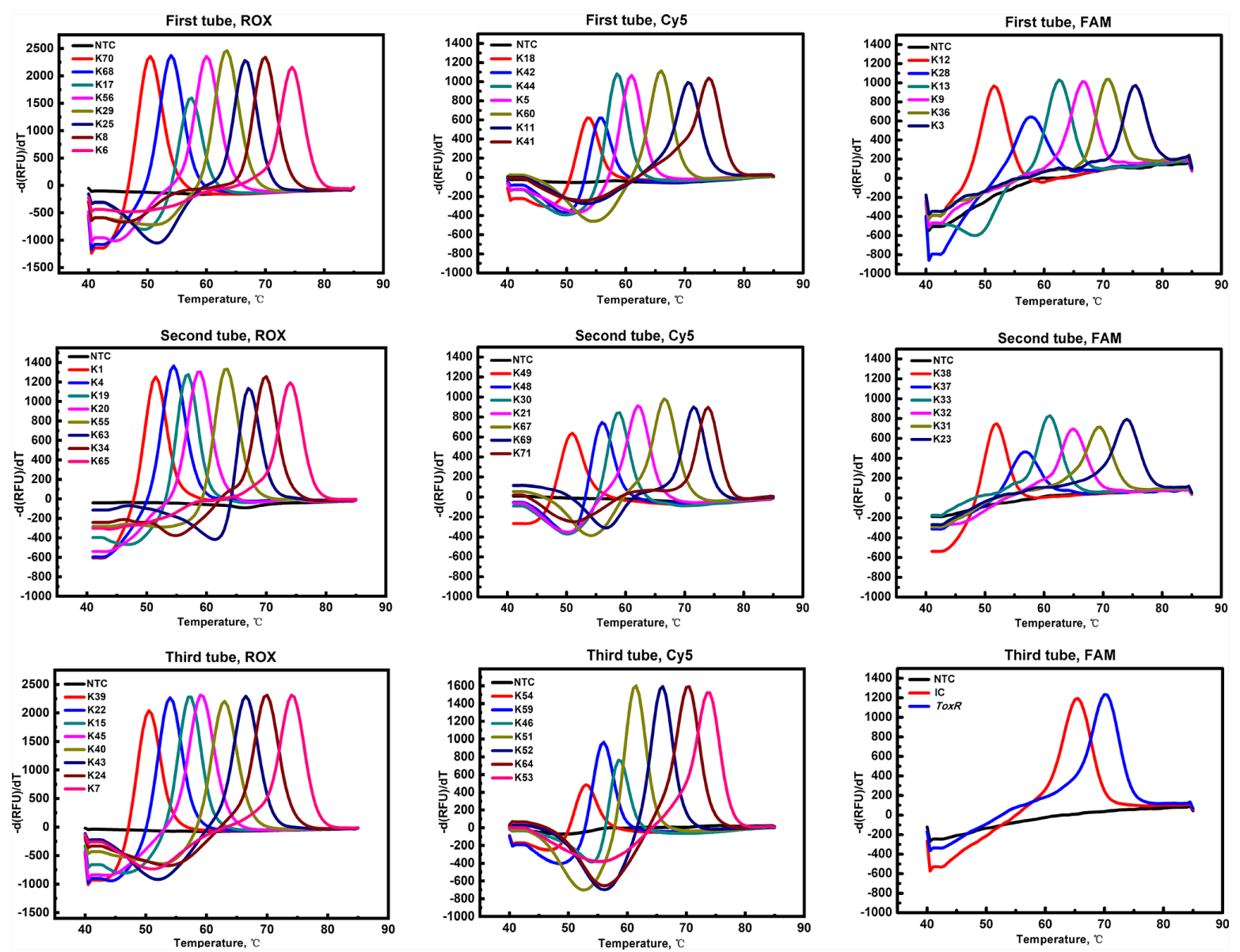

FIGURE 1 | Probe melting curve analysis for the identification of $V$. parahaemolyticus. $57 \mathrm{~K}$-serogroups in the multiplex ligation reaction based on probe melting curve analysis (MLMA) assay. Color-coded melting curves represent the different antigen genes in each fluorophore channel (ROX, FAM, and Cy5) in a three-tube system. IC, SUC2 gene was used as a positive internal control (IC); NTC, negative control. 
TABLE 4 | Reproducibility of designated melting temperatures (Tm) and Limit of Identification values for each serogroup target loci in the assay.

\begin{tabular}{|c|c|c|c|c|c|c|c|c|c|c|}
\hline \multirow[t]{2}{*}{ Name } & \multirow[t]{2}{*}{ Gene } & \multirow[t]{2}{*}{ Tube } & \multirow{2}{*}{$\begin{array}{l}\text { Concentration } \\
\qquad \mathrm{ng} / \mu \mathrm{l}\end{array}$} & \multicolumn{3}{|c|}{ Intra-assay reproducibility } & \multicolumn{3}{|c|}{ Inter-assay reproducibility } & \multirow{2}{*}{$\begin{array}{l}\text { Limit of identification } \\
\qquad(\mathrm{ng} / \mu \mathrm{l})\end{array}$} \\
\hline & & & & Mean $\operatorname{Tm}\left({ }^{\circ} \mathrm{C}\right)$ & SD & CV (\%) & Mean $\operatorname{Tm}\left({ }^{\circ} \mathrm{C}\right)$ & SD & CV (\%) & \\
\hline \multirow[t]{2}{*}{ K3 } & VP24500037 & 1 & 10.0 & 74.50 & 0.00 & 0.00 & 74.78 & 0.53 & 0.71 & 0.10 \\
\hline & & & 1.0 & 74.50 & 0.00 & 0.00 & 74.61 & 0.31 & 0.42 & \\
\hline \multirow[t]{2}{*}{ K5 } & VP19500014 & 1 & 10.0 & 61.00 & 0.00 & 0.00 & 61.00 & 0.24 & 0.39 & 0.10 \\
\hline & & & 1.0 & 61.00 & 0.00 & 0.00 & 61.00 & 0.00 & 0.00 & \\
\hline \multirow[t]{2}{*}{ K6 } & VP0223 & 1 & 10.0 & 74.50 & 0.00 & 0.00 & 74.44 & 0.16 & 0.21 & 0.10 \\
\hline & & & 1.0 & 74.17 & 0.24 & 0.32 & 74.22 & 0.25 & 0.33 & \\
\hline \multirow[t]{2}{*}{ K8 } & VPBB0234 & 1 & 10.0 & 70.00 & 0.00 & 0.00 & 69.78 & 0.25 & 0.36 & 0.10 \\
\hline & & & 1.0 & 69.50 & 0.00 & 0.00 & 69.50 & 0.24 & 0.34 & \\
\hline \multirow[t]{2}{*}{ K9 } & VP13500017 & 1 & 10.0 & 65.83 & 0.24 & 0.36 & 65.61 & 0.31 & 0.48 & 0.10 \\
\hline & & & 1.0 & 65.50 & 0.41 & 0.62 & 65.39 & 0.46 & 0.70 & \\
\hline K11 & VP10700010 & 1 & 10.0 & 70.33 & 0.24 & 0.34 & 70.33 & 0.24 & 0.34 & 0.10 \\
\hline & & & 1.0 & 70.50 & 0.00 & 0.00 & 70.44 & 0.16 & 0.22 & \\
\hline K12 & VP17900014 & 1 & 10.0 & 50.83 & 0.24 & 0.46 & 50.83 & 0.24 & 0.46 & 1.00 \\
\hline & & & 1.0 & 50.67 & 0.24 & 0.47 & 50.78 & 0.25 & 0.49 & \\
\hline K13 & VP9800011 & 1 & 10.0 & 61.33 & 0.24 & 0.38 & 61.67 & 0.47 & 0.76 & 0.10 \\
\hline & & & 1.0 & 61.17 & 0.24 & 0.39 & 61.17 & 0.24 & 0.39 & \\
\hline K17 & VP4300018 & 1 & 10.0 & 57.50 & 0.00 & 0.00 & 57.39 & 0.21 & 0.36 & 0.10 \\
\hline & & & 1.0 & 57.17 & 0.24 & 0.41 & 57.17 & 0.24 & 0.41 & \\
\hline K18 & VP19700016 & 1 & 10.0 & 53.50 & 0.00 & 0.00 & 53.61 & 0.21 & 0.39 & 0.10 \\
\hline & & & 1.0 & 53.83 & 0.24 & 0.44 & 53.72 & 0.25 & 0.46 & \\
\hline K25 & VP13200012 & 1 & 10.0 & 66.50 & 0.00 & 0.00 & 66.56 & 0.16 & 0.24 & 1.00 \\
\hline & & & 1.0 & 66.33 & 0.24 & 0.36 & 66.28 & 0.25 & 0.37 & \\
\hline K28 & VP5300035 & 1 & 10.0 & 57.00 & 0.00 & 0.00 & 57.33 & 0.47 & 0.82 & 0.10 \\
\hline & & & 1.0 & 56.83 & 0.24 & 0.41 & 56.83 & 0.24 & 0.41 & \\
\hline K29 & VP24700016 & 1 & 10.0 & 63.33 & 0.24 & 0.37 & 63.39 & 0.21 & 0.33 & 0.10 \\
\hline & & & 1.0 & 63.00 & 0.00 & 0.00 & 63.06 & 0.16 & 0.25 & \\
\hline K36 & VP12200010 & 1 & 10.0 & 69.83 & 0.24 & 0.34 & 69.78 & 0.34 & 0.49 & 0.10 \\
\hline & & & 1.0 & 69.83 & 0.24 & 0.34 & 69.83 & 0.33 & 0.48 & \\
\hline K41 & VP23400016 & 1 & 10.0 & 74.17 & 0.24 & 0.32 & 74.00 & 0.24 & 0.32 & 0.10 \\
\hline & & & 1.0 & 74.17 & 0.24 & 0.32 & 74.11 & 0.21 & 0.28 & \\
\hline K42 & VP400015 & 1 & 10.0 & 55.83 & 0.24 & 0.42 & 55.94 & 0.16 & 0.28 & 1.00 \\
\hline & & & 1.0 & 56.00 & 0.00 & 0.00 & 56.00 & 0.00 & 0.00 & \\
\hline K44 & VP11300018 & 1 & 10.0 & 58.50 & 0.00 & 0.00 & 58.50 & 0.00 & 0.00 & 0.10 \\
\hline & & & 1.0 & 58.50 & 0.00 & 0.00 & 58.50 & 0.00 & 0.00 & \\
\hline K56 & VP33400015 & 1 & 10.0 & 60.00 & 0.00 & 0.00 & 59.94 & 0.16 & 0.26 & 0.10 \\
\hline & & & 1.0 & 59.50 & 0.00 & 0.00 & 59.56 & 0.16 & 0.26 & \\
\hline K60 & VP1600020 & 1 & 10.0 & 66.00 & 0.00 & 0.00 & 65.94 & 0.16 & 0.24 & 0.10 \\
\hline & & & 1.0 & 66.00 & 0.00 & 0.00 & 66.06 & 0.16 & 0.24 & \\
\hline K68 & VP16100014 & 1 & 10.0 & 54.00 & 0.00 & 0.00 & 54.06 & 0.16 & 0.29 & 0.10 \\
\hline & & & 1.0 & 54.00 & 0.00 & 0.00 & 53.94 & 0.16 & 0.29 & \\
\hline K70 & $w z y$ & 1 & 10.0 & 50.50 & 0.00 & 0.00 & 50.44 & 0.16 & 0.31 & 0.10 \\
\hline & & & 1.0 & 50.33 & 0.24 & 0.47 & 50.39 & 0.21 & 0.41 & \\
\hline K1 & VP30500018 & 2 & 10.0 & 50.83 & 0.29 & 0.57 & 50.83 & 0.25 & 0.49 & 0.10 \\
\hline & & & 1.0 & 50.33 & 0.29 & 0.57 & 50.67 & 0.35 & 0.70 & \\
\hline K4 & VP31900014 & 2 & 10.0 & 54.00 & 0.00 & 0.00 & 54.00 & 0.00 & 0.00 & 0.10 \\
\hline & & & 1.0 & 53.50 & 0.00 & 0.00 & 53.72 & 0.26 & 0.49 & \\
\hline K19 & VP19200012 & 2 & 10.0 & 57.00 & 0.00 & 0.00 & 56.89 & 0.22 & 0.39 & 0.10 \\
\hline & & & 1.0 & 56.33 & 0.29 & 0.51 & 56.50 & 0.35 & 0.63 & \\
\hline K20 & VP20600013 & 2 & 10.0 & 59.00 & 0.00 & 0.00 & 59.00 & 0.00 & 0.00 & 0.10 \\
\hline & & & 1.0 & 58.67 & 0.29 & 0.49 & 58.67 & 0.25 & 0.43 & \\
\hline $\mathrm{K} 21$ & VP43900016 & 2 & 10.0 & 62.00 & 0.00 & 0.00 & 61.83 & 0.25 & 0.40 & 1.00 \\
\hline & & & 1.0 & 61.00 & 0.00 & 0.00 & 61.17 & 0.25 & 0.41 & \\
\hline K23 & VP20200014 & 2 & 10.0 & 74.00 & 0.00 & 0.00 & 74.17 & 0.25 & 0.34 & 0.10 \\
\hline & & & 1.0 & 74.00 & 0.00 & 0.00 & 74.28 & 0.36 & 0.49 & \\
\hline K30 & VP19000009 & 2 & 10.0 & 58.67 & 0.29 & 0.49 & 58.61 & 0.22 & 0.38 & 0.10 \\
\hline & & & 1.0 & 58.50 & 0.00 & 0.00 & 58.50 & 0.00 & 0.00 & \\
\hline K31 & VP20500017 & 2 & 10.0 & 69.50 & 0.00 & 0.00 & 69.67 & 0.25 & 0.36 & 1.00 \\
\hline & & & 1.0 & 69.50 & 0.00 & 0.00 & 69.72 & 0.26 & 0.38 & \\
\hline K32 & VP9900015 & 2 & 10.0 & 65.50 & 0.00 & 0.00 & 65.72 & 0.26 & 0.40 & 0.10 \\
\hline & & & 1.0 & 65.67 & 0.29 & 0.44 & 65.83 & 0.35 & 0.54 & \\
\hline K33 & VP20100010 & 2 & 10.0 & 61.00 & 0.00 & 0.00 & 61.11 & 0.33 & 0.55 & 0.10 \\
\hline & & & 1.0 & 61.17 & 0.29 & 0.47 & 61.17 & 0.35 & 0.58 & \\
\hline
\end{tabular}


TABLE 4 | Continued

\begin{tabular}{|c|c|c|c|c|c|c|c|c|c|c|}
\hline \multirow[t]{2}{*}{ Name } & \multirow[t]{2}{*}{ Gene } & \multirow[t]{2}{*}{ Tube } & \multirow{2}{*}{$\begin{array}{c}\text { Concentration } \\
\qquad \mathrm{ng} / \mu \mathrm{l}\end{array}$} & \multicolumn{3}{|c|}{ Intra-assay reproducibility } & \multicolumn{3}{|c|}{ Inter-assay reproducibility } & \multirow{2}{*}{$\begin{array}{l}\text { Limit of identification } \\
\qquad(\mathrm{ng} / \mu \mathrm{l})\end{array}$} \\
\hline & & & & Mean $\mathrm{Tm}\left({ }^{\circ} \mathrm{C}\right)$ & SD & CV (\%) & Mean $\operatorname{Tm}\left({ }^{\circ} \mathrm{C}\right)$ & SD & CV (\%) & \\
\hline \multirow[t]{2}{*}{ K34 } & VP1500017 & 2 & 10.0 & 69.83 & 0.29 & 0.41 & 69.94 & 0.17 & 0.24 & 0.10 \\
\hline & & & 1.0 & 70.00 & 0.00 & 0.00 & 69.89 & 0.22 & 0.32 & \\
\hline \multirow[t]{2}{*}{ K37 } & VP23900011 & 2 & 10.0 & 57.00 & 0.00 & 0.00 & 57.17 & 0.25 & 0.44 & 1.00 \\
\hline & & & 1.0 & 56.67 & 0.29 & 0.51 & 56.72 & 0.26 & 0.46 & \\
\hline \multirow[t]{2}{*}{ K38 } & VP20000015 & 2 & 10.0 & 51.67 & 0.29 & 0.56 & 51.83 & 0.25 & 0.48 & 0.10 \\
\hline & & & 1.0 & 51.50 & 0.00 & 0.00 & 51.56 & 0.30 & 0.58 & \\
\hline \multirow[t]{2}{*}{ K48 } & VP22900017 & 2 & 10.0 & 55.83 & 0.29 & 0.52 & 55.61 & 0.22 & 0.40 & 0.10 \\
\hline & & & 1.0 & 55.50 & 0.00 & 0.00 & 55.61 & 0.22 & 0.40 & \\
\hline \multirow[t]{2}{*}{ K49 } & VP23800014 & 2 & 10.0 & 50.50 & 0.00 & 0.00 & 50.44 & 0.17 & 0.33 & 0.10 \\
\hline & & & 1.0 & 50.17 & 0.29 & 0.58 & 50.33 & 0.25 & 0.50 & \\
\hline \multirow[t]{2}{*}{ K55 } & VP100017 & 2 & 10.0 & 62.50 & 0.00 & 0.00 & 62.67 & 0.25 & 0.40 & 0.10 \\
\hline & & & 1.0 & 62.50 & 0.00 & 0.00 & 62.67 & 0.25 & 0.40 & \\
\hline \multirow[t]{2}{*}{ K63 } & VP3400018 & 2 & 10.0 & 66.50 & 0.00 & 0.00 & 66.50 & 0.00 & 0.00 & 0.10 \\
\hline & & & 1.0 & 66.33 & 0.29 & 0.44 & 66.44 & 0.17 & 0.25 & \\
\hline \multirow[t]{2}{*}{ K65 } & $w z y$ & 2 & 10.0 & 73.50 & 0.00 & 0.00 & 73.67 & 0.25 & 0.34 & 0.10 \\
\hline & & & 1.0 & 73.50 & 0.00 & 0.00 & 73.72 & 0.26 & 0.36 & \\
\hline K67 & $w z y$ & 2 & 10.0 & 66.50 & 0.00 & 0.00 & 66.17 & 0.25 & 0.38 & 0.10 \\
\hline & & & 1.0 & 65.67 & 0.29 & 0.44 & 65.72 & 0.26 & 0.40 & \\
\hline K69 & VP3300015 & 2 & 10.0 & 71.50 & 0.00 & 0.00 & 71.00 & 0.43 & 0.61 & 0.10 \\
\hline & & & 1.0 & 70.33 & 0.29 & 0.41 & 70.50 & 0.25 & 0.35 & \\
\hline K71 & VP3200012 & 2 & 10.0 & 74.50 & 0.00 & 0.00 & 74.56 & 0.17 & 0.22 & 0.10 \\
\hline & & & 1.0 & 73.67 & 0.29 & 0.39 & 73.67 & 0.25 & 0.34 & \\
\hline K7 & $w z y$ & 3 & 10.0 & 74.00 & 0.00 & 0.00 & 73.94 & 0.16 & 0.21 & 0.10 \\
\hline & & & 1.0 & 73.83 & 0.24 & 0.32 & 73.78 & 0.25 & 0.34 & \\
\hline K15 & $w z y$ & 3 & 10.0 & 57.17 & 0.24 & 0.41 & 57.11 & 0.21 & 0.36 & 0.10 \\
\hline & & & 1.0 & 57.17 & 0.24 & 0.41 & 57.11 & 0.21 & 0.36 & \\
\hline K22 & $w z x$ & 3 & 10.0 & 54.00 & 0.00 & 0.00 & 54.00 & 0.00 & 0.00 & 0.10 \\
\hline & & & 1.0 & 53.83 & 0.24 & 0.44 & 53.78 & 0.25 & 0.46 & \\
\hline K24 & $w z y$ & 3 & 10.0 & 70.00 & 0.00 & 0.00 & 70.00 & 0.00 & 0.00 & 0.10 \\
\hline & & & 1.0 & 70.00 & 0.41 & 0.58 & 70.00 & 0.33 & 0.48 & \\
\hline K39 & wzy & 3 & 10.0 & 50.50 & 0.00 & 0.00 & 50.56 & 0.16 & 0.31 & 0.10 \\
\hline & & & 1.0 & 50.33 & 0.24 & 0.47 & 50.39 & 0.39 & 0.78 & \\
\hline K40 & wzy & 3 & 10.0 & 62.50 & 0.00 & 0.00 & 62.72 & 0.25 & 0.40 & 0.10 \\
\hline & & & 1.0 & 62.67 & 0.24 & 0.38 & 62.83 & 0.33 & 0.53 & \\
\hline K43 & wzy & 3 & 10.0 & 66.50 & 0.00 & 0.00 & 66.50 & 0.00 & 0.00 & 0.10 \\
\hline & & & 1.0 & 66.33 & 0.24 & 0.36 & 66.39 & 0.21 & 0.31 & \\
\hline K45 & wzy & 3 & 10.0 & 59.00 & 0.00 & 0.00 & 59.06 & 0.16 & 0.27 & 0.10 \\
\hline & & & 1.0 & 59.17 & 0.24 & 0.40 & 59.06 & 0.28 & 0.48 & \\
\hline K46 & $w z y$ & 3 & 10.0 & 58.67 & 0.24 & 0.40 & 58.67 & 0.24 & 0.40 & 0.10 \\
\hline & & & 1.0 & 58.67 & 0.24 & 0.40 & 58.61 & 0.31 & 0.54 & \\
\hline K51 & $w z y$ & 3 & 10.0 & 61.50 & 0.00 & 0.00 & 61.44 & 0.16 & 0.26 & 0.10 \\
\hline & & & 1.0 & 61.33 & 0.24 & 0.38 & 61.28 & 0.25 & 0.41 & \\
\hline K52 & $w Z x$ & 3 & 10.0 & 66.00 & 0.00 & 0.00 & 65.94 & 0.16 & 0.24 & 0.10 \\
\hline & & & 1.0 & 65.83 & 0.24 & 0.36 & 65.72 & 0.34 & 0.52 & \\
\hline K53 & wzy & 3 & 10.0 & 74.00 & 0.00 & 0.00 & 73.78 & 0.25 & 0.34 & 0.10 \\
\hline & & & 1.0 & 73.83 & 0.24 & 0.32 & 73.78 & 0.25 & 0.34 & \\
\hline K54 & $w z y$ & 3 & 10.0 & 53.00 & 0.00 & 0.00 & 53.00 & 0.00 & 0.00 & 0.10 \\
\hline & & & 1.0 & 53.00 & 0.00 & 0.00 & 53.00 & 0.00 & 0.00 & \\
\hline K59 & $w z y$ & 3 & 10.0 & 56.00 & 0.00 & 0.00 & 56.00 & 0.00 & 0.00 & 0.10 \\
\hline & & & 1.0 & 56.00 & 0.00 & 0.00 & 56.00 & 0.00 & 0.00 & \\
\hline K64 & wzy & 3 & 10.0 & 70.50 & 0.00 & 0.00 & 70.39 & 0.21 & 0.30 & 0.10 \\
\hline & & & 1.0 & 70.33 & 0.24 & 0.34 & 70.33 & 0.24 & 0.34 & \\
\hline IC & SUC2 & 3 & 10.0 & 65.50 & 0.00 & 0.00 & 65.06 & 0.37 & 0.57 & 0.10 \\
\hline & & & 1.0 & 65.17 & 0.47 & 0.72 & 64.94 & 0.37 & 0.57 & \\
\hline VP & toxR & 3 & 10.0 & 70.17 & 0.24 & 0.34 & 69.72 & 0.48 & 0.69 & 0.10 \\
\hline & & & 1.0 & 69.83 & 0.24 & 0.34 & 69.67 & 0.47 & 0.68 & \\
\hline
\end{tabular}

outbreaks and can provide historical and comparable data for the surveillance of $V$. parahaemolyticus infections on a global scale (Han et al., 2016; Baker-Austin et al., 2018). Thus, the development of rapid and robust serotyping methods can facilitate national and international surveillance of $V$. parahaemolyticus infections.

In this study, we developed a molecular assay for the simultaneous identification of $57 \mathrm{~V}$. parahaemolyticus K- 
TABLE 5 | The results of serotype identification by multiplex ligation reaction based on probe melting curve analysis (MLMA) assay and conventional serotyping tests among $V$. parahaemolyticus isolates $(n=595)$ from the double-blind study.

\begin{tabular}{|c|c|c|c|c|c|c|c|}
\hline \multirow[t]{2}{*}{ MLMA } & \multicolumn{2}{|c|}{ Conventional serotyping } & \multirow[t]{2}{*}{ Total } & \multirow[t]{2}{*}{ Sensitivity } & \multirow[t]{2}{*}{ Specificity } & \multirow[t]{2}{*}{ Consistency rate } & \multirow[t]{2}{*}{ Kappa value } \\
\hline & + & - & & & & & \\
\hline+ & 359 & 18 & 377 & & & & \\
\hline- & 0 & 218 & 218 & $100 \%$ & $92.37 \%$ & $96.97 \%$ & $0.936(P=0.00)$ \\
\hline Total & 359 & 236 & 595 & & & & \\
\hline
\end{tabular}

MLMA (+) and Conventional serotyping (+) indicates that the results of both tests are of the same specific $K$-serogroups.

MLMA (+) and Conventional serotyping (-) indicates that the former results are specific $K$-serogroups and the latter are KUT.

MLMA (-) and Conventional serotyping (-) indicates that the results of both tests are KUT.

MLMA (-) and Conventional serotyping (+) indicates that the former results are KUT and the latter are specific K-serogroups.

serogroups based on the principles of MLMA. The assay can detect all gene targets with expected Tm values and no cross-reaction was observed. The detection limit of the assay ranged from 0.1 to $1.0 \mathrm{ng} /$ $\mu \mathrm{l}$. Moreover, the intra-assay and inter-assay reproducibility analyses showed that the assay had highly reproducible traits, with the largest SD value for the mean Tm being no more than $1{ }^{\circ} \mathrm{C}$ and the CVs being less than $1 \%$. Overall, the MLMA assay demonstrated a $96.97 \%$ consistency rate with the conventional serological method of all isolates and a kappa value of 0.936 , which indicated the consistency of the two approaches is excellent.

With the growing number of genomic sequences and serogroup-specific gene sequences available in public databases, various molecular serotyping methods have been developed as alternatives for serotyping, such as common and real-time PCR, multiplex PCR-based microarrays, Luminex-based serotyping assays and sequencing-based approaches (Liu et al., 2015; CabralCastro et al., 2016; Afroj et al., 2017; Pang et al., 2019). Although crucial progress regarding antigen synthesis gene clusters and serogroup-specific genes for Salmonella and Escherichia coli, there has been little progress on the capsular polysaccharide gene clusters or genetic targets of $V$. parahaemolyticus $\mathrm{K}$-antigens to date. Thus, the development of a molecular method for $V$. parahaemolyticus $\mathrm{K}$ serogroups is highly dependent on the investigation of the genetic characteristics of $V$. parahaemolyticus K-antigen. Early studies of Kantigen genetic determinants in $V$. parahaemolyticus produced controversial results (Guvener and Mccarter, 2003 Okura et al., 2008). Later, in 2010, the detailed genetic determinants of K-antigen synthesis were confirmed to be between gmhD and rig using an $\mathrm{O} 3$ : K6 isolate based on the construction of gene deletions (Chen et al., 2010). We used the whole genome sequence of $418 \mathrm{~V}$. parahaemolyticus strains to design the target genes primers and probes and then analyzed the genetic structure and evolutionary relationship of their $39 \mathrm{~K}$-serogroups to identify the serogroupspecific genes of the capsular polysaccharide gene clusters (Bian et al., 2021). Additionally, another capsular polysaccharide gene clusters of 18 rare K-serogroups that we lack these data were analyzed (Pang et al., 2019). Thus, $57 \mathrm{~K}$-serogroups specific sequences were selected for the development of $V$. parahaemolyticus serotyping assay.

Currently, only three assays have the ability to detect more than two $V$. parahaemolyticus K-serogroups, the first is a microspherebased suspension array, the second is the sequence-based serotyping of $V$. parahaemolyticus $55 \mathrm{~K}$-serogroups, and the third is the MLMA assay we presented for the simultaneous identification of nine K-serogroups (Li et al., 2019; Pang et al.,
2019). However, the microsphere-based suspension array and sequence-based serotyping method require expensive equipment with related software and rely on bioinformatics analysis. In this study, we expanded the high throughput targets of our previously developed MLMA assay from nine K-serogroups to $57 \mathrm{~K}$ serogroups and identified five rare $\mathrm{K}$-serogroups using the method. To date, we have been able to detect $12 \mathrm{O}$-serogroups (Li et al., 2019) and $57 \mathrm{~K}$-serogroups in four tubes using the established MLMA assay.

Upon the evaluation of the assay using 595 isolates over 15 years (2003-2018) from the Shenzhen Center for Disease Control and Prevention, the MLMA assay accurately detected 377 typeable isolates that belonged to $29 \mathrm{~K}$-serogroups and 218 untypeable isolates. In addition, there were inconsistent results for 18 isolates generated by the MLMA assay and the serological method. The results of Sanger sequencing of the PCR amplicons of these isolates were consistent with the results of the MLMA assay, which indicates the MLMA assay has higher sensitivity and accuracy than the serological method. Among 377 typeable isolates, five rare Kserogroups were detected (2008-2017) by the MLMA assay, which suggested that $V$. parahaemolyticus with extremely rare Kserogroups emerged in Shenzhen as early as 2008, and that routine monitoring should be conducted. The current assay showed similar superior performances of the assay as previously reported. However, the entire MLMA assay could be completed within 3.5-4.0 h, which saves 12-18 h when compared with traditional serotyping (Li et al., 2019). Additionally, a simple boiled lysates protocol is used for the preparation of DNA templates, which also increases the flexibility of the MLMA assay. As demonstrated by the analytical studies, the MLMA assay could accurately and reproducibly detect $57 \mathrm{~V}$. parahaemolyticus K-serogroups. Hence, the targets of the assay covered approximately $81 \% \mathrm{~V}$. parahaemolyticus K-serogroups that have been identified worldwide, demonstrating that multiplex assays facilitate timely and effective detection.

However, this assay was unable to differentiate two closely related K-serogroup pairs, K56 and K57, K47, and K48, which could be attributed to their capsular polysaccharide gene clusters showing almost identical homology to the corresponding regions (Pang et al., 2019). Currently, genetic markers for the accurate identification of these two pairs are unavailable and some strains were still untypeable. Therefore, further research is necessary to enable differentiation of these serogroups at the molecular level. Furthermore, with the development of whole-genome sequencing, the increasing demand for the sequence-based serotyping method gives us an important 
consideration to combine the MLMA assay with the genotyping for more effective foodborne disease outbreak investigation, source tracing, and the surveillance of $V$. parahaemolyticus infection.

In a word, a novel molecular assay was developed for simultaneous detection of $57 \mathrm{~K}$-serogroups of $\mathrm{V}$. parahaemolyticus and five rare $\mathrm{K}$-serogroups were found. This assay can provide a rapid, accurate, and highly sensitive identification of $V$. parahaemolyticus $\mathrm{K}$-serogroups and is valuable for screening large samples during vibriosis outbreaks and surveillance.

\section{DATA AVAILABILITY STATEMENT}

The datasets presented in this study can be found in online repositories. The names of the repository/repositories and accession number(s) can be found in the article/Supplementary Material (Table S8 and Table S9).

\section{AUTHOR CONTRIBUTIONS}

LLu and ML conceived and designed the experiments. LLu and ML performed the experiments and contributed to analysis. LLu, $\mathrm{ML}$, and QH wrote the paper. YLi, MJ, and XS guided the selection of experimental strains. YQ and RC checked the information of

\section{REFERENCES}

Afroj, S., Aldahami, K., Reddy, G., Guard, J., Adesiyun, A., Samuel, T., et al. (2017). Simultaneous detection of multiple Salmonella serovars from milk and chicken meat by real-time PCR using unique genomic target regions. J. Food Prot. 80, 1944-1957. doi: 10.4315/0362-028X.JFP-17-133

Baker-Austin, C., Oliver, J. D., Alam, M., Ali, A., Waldor, M. K., Qadri, F., et al. (2018). Vibrio spp. infections. Nat. Rev. Dis. Primers 4, 8. doi: 10.1038/s41572018-0005-8

Bhuiyan, N. A., Ansaruzzaman, M., Kamruzzaman, M., Alam, K., Chowdhury, N. R., Nishibuchi, M., et al. (2002). Prevalence of the Pandemic Genotype of Vibrio parahaemolyticus in Dhaka, Bangladesh, and Significance of Its Distribution across Different Serotypes. J. Clin. Microbiol. 40, 284-286. doi: 10.1128/JCM.40.1.284286.2002

Bian, S., Wenhong, Z., Li, Q., Li, Y., Wong, N.-K., Jiang, M., et al. (2021). Genetic structure, function and evolution of capsule biosynthesis loci in Vibrio parahaemolyticus. Front. Microbiol. 11, 3218. doi: 10.1101/2020.02.25. 964247

Cabral-Castro, M. J., Peralta, R. H. S., Cavalcanti, M. G., Puccioni-Sohler, M., Carvalho, V. L., Da Costa Vasconcelos, P. F., et al. (2016). A Luminex-based single DNA fragment amplification assay as a practical tool for detecting and serotyping dengue virus. J. Virol. Methods 236, 18-24. doi: 10.1016/ j.jviromet.2016.07.003

Chen, Y., Dai, J., Morris, J. G. Jr., and Johnson, J. A. (2010). Genetic analysis of the capsule polysaccharide ( $\mathrm{K}$ antigen) and exopolysaccharide genes in pandemic Vibrio parahaemolyticus O3:K6. BMC Microbiol. 10, 274. doi: 10.1186/14712180-10-274

Chen, M., Guo, D., Wong, H. C., Zhang, X., Liu, F., Chen, H., et al. (2012). Development of O-serogroup specific PCR assay for detection and identification of Vibrio parahaemolyticus. Int. J. Food Microbiol. 159, 122129. doi: 10.1016/j.ijfoodmicro.2012.08.012

Chen, X., Li, Y., Yao, W., Wu, T., Zhu, Q., Zhang, Y., et al. (2020). A new emerging serotype of Vibrio parahaemolyticus in China is rapidly becoming the main epidemic strain. Clin. Microbiol. Infect. 26, 644.e1-644.e7. doi: 10.2139/ ssrn.3294765

Fu, S., Wei, D., Yang, Q., Xie, G., Pang, B., Wang, Y., et al. (2020). Horizontal Plasmid Transfer Promotes the Dissemination of Asian Acute strains. YJ, LZ, and LW guided the experimental operation. YL and QL provided experimental technical support. SB and LLi analyzed the gene sequence. $\mathrm{LLu}$ and $\mathrm{ML}$ contributed equally to this article. All authors contributed to the article and approved the submitted version.

\section{FUNDING}

This research was supported by the China National Science and Technology Major Projects Foundation (No. 2017ZX10303406), National Natural Science Foundation of China (No. 81773436), Sanming Project of Medicine in Shenzhen (No. SZSM201811071), Shenzhen Key Medical Discipline Construction Fund (SZXK064), and the Shenzhen Health and Family Planning Commission (SZGW2017004).

\section{SUPPLEMENTARY MATERIAL}

The Supplementary Material for this article can be found online at: https://www.frontiersin.org/articles/10.3389/fcimb.2021. 594808/full\#supplementary-material

Hepatopancreatic Necrosis Disease and Provides a Novel Mechanism for Genetic Exchange and Environmental Adaptation. mSystems 5, e00799e00719. doi: 10.1128/mSystems.00799-19

Guvener, Z. T., and Mccarter, L. L. (2003). Multiple Regulators Control Capsular Polysaccharide Production in Vibrio parahaemolyticus. J. Bacteriol. 185, 54315441. doi: 10.1128/JB.185.18.5431-5441.2003

Han, C., Tang, H., Ren, C., Zhu, X., and Han, D. (2016). Sero-Prevalence and Genetic Diversity of Pandemic V. parahaemolyticus Strains Occurring at a Global Scale. Front. Microbiol. 7, 567. doi: 10.3389/fmicb.2016.00567

Han, D., Yu, F., Chen, X., Zhang, R., and Li, J. (2019). Challenges in Vibrio parahaemolyticus infections caused by the pandemic clone. Future Microbiol. 14, 437-450. doi: 10.2217/fmb-2018-0308

Iguchi, T., Kondo, S., and Hisatsune, K. (1995). Vibrio parahaemolyticus O serotypes from 01 to 013 all produce R-type lipopolysaccharide:SDS-PAGE and compositional sugar analysis. FEMS Microbiol. Lett. 130, 287-292. doi: 10.1111/j.1574-6968.1995.tb07733.x

Jiang, Y., He, L., Wu, P., Shi, X., Jiang, M., Li, Y., et al. (2017). Simultaneous Identification of Ten Bacterial Pathogens Using the Multiplex Ligation Reaction Based on the Probe Melting Curve Analysis. Sci. Rep. 7, 5902. doi: 10.1038/s41598-017-06348-z

Lai, H. C., Ng, T. H., Ando, M., Lee, C. T., Chen, I. T., Chuang, J. C., et al. (2015). Pathogenesis of acute hepatopancreatic necrosis disease (AHPND) in shrimp. Fish Shellfish Immunol. 47, 1006-1014. doi: 10.1016/j.fsi.2015.11.008

Letchumanan, V., Chan, K. G., and Lee, L. H. (2014). Vibrio parahaemolyticus: a review on the pathogenesis, prevalence, and advance molecular identification techniques. Front. Microbiol. 5, 705. doi: 10.3389/fmicb.2014.00705

Li, P., Xin, W., Xia, S., Luo, Y., Chen, Z., Jin, D., et al. (2018). MALDI-TOF mass spectrometry-based serotyping of $\mathrm{V}$. parahaemolyticus isolated from the Zhejiang province of China. BMC Microbiol. 18, 185. doi: 10.1186/s12866-018-1328-Z

Li, M., Jiang, Y., Shi, X., Li, Y., Jiang, M., Lin, Y., et al. (2019). Simultaneous Identification of Clinically Common Vibrio parahaemolyticus Serotypes Using Probe Melting Curve Analysis. Front. Cell. Infect. Microbiol. 14, 385. doi: 10.3389/fcimb.2019.00385

Liao, Y., Wang, X., Sha, C., Xia, Z., Huang, Q., and Li, Q. (2013). Combination of fluorescence color and melting temperature as a two-dimensional label for homogeneous multiplex PCR detection. Nucleic Acids Res. 41, e76. doi: 10.1093/nar/gkt004 
Liu, Y., Yan, X., Debroy, C., Fratamico, P. M., Needleman, D. S., Li, R. W., et al. (2015). Escherichia coli O-antigen gene clusters of serogroups O62, O68, O131, O140, O142, and O163: DNA sequences and similarity between O62 and O68, and PCR-based serogrouping. Biosensors (Basel) 5, 51-68. doi: 10.3390/ bios 5010051

Liu, J., Bai, L., Li, W., Han, H., Fu, P., Ma, X., et al. (2018). Trends of foodborne diseases in China: lessons from laboratory-based surveillance since 2011. Front. Med. 12, 48-57. doi: 10.1007/s11684-017-0608-6

Matsumoto, C., Okuda, J., Ishibashi, M., and Bashi, (2000). Pandemic spread of an O3:K6 clone of Vibrio parahaemolyticus and emergence of related strains evidenced by arbitrarily primed PCR and toxRS sequence analyses. J. Clin. Microbiol. 38, 578-585. doi: 10.1128/JCM.38.2.578585.2000

Nair, G. B., Ramamurthy, T., Bhattacharya, S. K., Dutta, B., Takeda, Y., and Sack, D. A. (2007). Global dissemination of Vibrio parahaemolyticus serotype O3:K6 and its serovariants. Clin. Microbiol. Rev. 20, 39-48. doi: 10.1128/ CMR.00025-06

Okura, M., Osawa, R., Tokunaga, A., Morita, M., Arakawa, E., and Watanabe, H. (2008). Genetic analyses of the putative $\mathrm{O}$ and $\mathrm{K}$ antigen gene clusters of pandemic Vibrio parahaemolyticus. Microbiol. Immunol. 52, 251-264. doi: 10.1111/j.1348-0421.2008.00027.x

Pang, Y., Guo, X., Tian, X., Liu, F., Wang, L., Wu, J., et al. (2019). Developing a novel molecular serotyping system based on capsular polysaccharide synthesis gene clusters of Vibrio parahaemolyticus. Int. J. Food Microbiol. 309, 108332. doi: 10.1016/j.ijfoodmicro.2019.108332

Wang, R., Zhong, Y., Gu, X., Yuan, J., Saeed, A. F., and Wang, S. (2015). The pathogenesis, detection, and prevention of Vibrio parahaemolyticus. Front. Microbiol. 6, 144. doi: 10.3389/fmicb.2015.00144

Zuo, L., Jiang, M., Jiang, Y., Shi, X., Li, Y., Lin, Y., et al. (2019). Multiplex ligation reaction based on probe melting curve analysis: a pragmatic approach for the identification of 30 common Salmonella serovars. Ann. Clin. Microbiol. Antimicrob. 18, 39. doi: 10.1186/s12941-019-0338-5

Conflict of Interest: SB and LLi was employed by BGI-Shenzhen.

The remaining authors declare that the research was conducted in the absence of any commercial or financial relationships that could be construed as a potential conflict of interest.

Copyright (c) $2021 \mathrm{Lu}$, Li, Li, Jiang, Jiang, Shi, Zuo, Wang, Bian, Qiu, Cai, Liao, Li, Li and $\mathrm{Hu}$. This is an open-access article distributed under the terms of the Creative Commons Attribution License (CC BY). The use, distribution or reproduction in other forums is permitted, provided the original author(s) and the copyright owner(s) are credited and that the original publication in this journal is cited, in accordance with accepted academic practice. No use, distribution or reproduction is permitted which does not comply with these terms. 